\title{
Ultranarrow homogeneous broadening of confined excitons in quantum dots: Effect of the surrounding matrix
}

\author{
Michio Ikezawa and Yasuaki Masumoto \\ Institute of Physics, University of Tsukuba, Tsukuba, Ibaraki 305-8571, Japan
}

(Received 20 December 1999)

\begin{abstract}
Ultranarrow homogeneous linewidth of a $Z_{3}$ exciton in $\mathrm{CuCl}$ quantum dots was studied by using accumulated photon echo. $\mathrm{CuCl}$ quantum dots embedded in two kinds of matrices, namely, an alminoborosilicate glass and a $\mathrm{NaCl}$ crystal, were studied. The narrowest homogeneous linewidth at low temperature was $1 \mu \mathrm{eV}$. The temperature dependence of the homogeneous linewidth was similar, above $5 \mathrm{~K}$ for both kinds of samples, but in the lower-temperature region, a remarkable difference was observed between them. It indicates that the matrix definitely affects the dephasing process of a confined exciton in quantum dots at low temperatures. The observed temperature dependence was explained by the contribution of the confined phonon in quantum dots and the two-level system in the matrix.
\end{abstract}

Recently, semiconductor quantum dots have received considerable attention because of their unique optical properties and their possibility in optical device applications. In quantum dots, atomiclike discrete energy levels of the electronic states are expected by the three-dimensional confinement of the electronic wave functions. ${ }^{1}$ The linewidth of the transitions between these quantized levels is not zero, but is finite, reflecting their broadening mechanisms. As possible broadening mechanisms of quantum dots, lifetime broadening, defect or impurity scattering, surface scattering, phonon scattering, and carrier-carrier scattering can be considered. The full linewidth at the half maximum (FWHM) of the homogeneous spectrum, $\Gamma_{h}$, is proportional to the inverse of the dephasing time $\left(T_{2}\right)$, which is the sum of the dephasing rates of these mechanisms of the related energy levels, as $\Gamma_{h}=2 \hbar / T_{2}$. Roughly speaking, the above-stated mechanisms are intrinsic to the quantum dots. In other words, they are also applicable to quantum dots floated in vacuum. However, most of the quantum dots are not free-standing, but are surrounded by matrices of larger band-gap energy, such as quantum dots embedded in a glass matrix. These circumstances give some complexity to the quantum dots. As a result, matrix or surface related interesting phenomena such as persistent spectral hole burning (PSHB), ${ }^{2}$ photodarkening, ${ }^{3}$ and fluorescence intermittency ${ }^{4}$ are observed in quantum dots. In this way, the physics of quantum dots being inseparable from the influence of the matrix, it is likely that the matrix affects the homogeneous linewidth of quantum dots. In fact, an atom (ion) embedded in host crystals as an impurity is well known to show broader linewidth because of the perturbation of the host crystal, and its broadening mechanisms have been studied extensively. However, quantum dots - they are often referred to as artificial atoms-to our knowledge, have never been studied in this direction. In this paper, the homogeneous linewidth is measured experimentally by using $\mathrm{CuCl}$ quantum dots as a sample where the linewidth in the bulk material is well studied, ${ }^{5-7}$ and its broadening mechanism is discussed by paying attention to the influence of the matrix.

Many quantum dot systems show inhomogeneous broadening because of the size and environment distribution of the dots. To measure the homogeneous spectrum buried in the inhomogeneously broadened spectrum, site-selective excitation techniques ${ }^{8,9}$ or single quantum dot spectroscopy ${ }^{10,11}$ has been applied. In consequence, the homogeneous linewidth of many quantum dot systems was found to be very sharp $(\leqslant 0.1 \mathrm{meV})$. Therefore, it seems to be difficult to evaluate the homogeneous width correctly by using a grating spectrometer, whose spectral resolution is typically about 0.1 $\mathrm{meV}$. On the other hand, time domain measurements such as photon echo are useful in this case. In the present work, we measured the dephasing time of a $Z_{3}$ exciton confined in $\mathrm{CuCl}$ quantum dots (QD's) by using heterodyne-detected accumulated photon echo method. ${ }^{12}$ By applying this technique, very low input power can be used to avoid the inputpower-dependent broadening, and there is no limit on the spectral resolution, since a sharp linewidth corresponds to a long dephasing time. Very recently, there were two reports on the measurement of the dephasing time of $\mathrm{CuCl}$ QD's. ${ }^{13,14}$ However, as the dephasing mechanism was not understood, we considered that more elaborate experimental work toward lower temperatures including a change of the matrix was necessary for its understanding.

Experimental setup is almost the same as was used in Ref. 12. The light source was a picosecond mode-locked Ti:sapphire laser, whose typical pulse width and repetition rate were $2 \mathrm{ps}$ and $82 \mathrm{MHz}$, respectively. We used two kind of samples, namely, $\mathrm{CuCl}$ quantum dots embedded in a $\mathrm{NaCl}$ crystal and $\mathrm{CuCl}$ quantum dots in alminoborosilicate glass, referred to as $\mathrm{CuCl} / \mathrm{NaCl}$ and $\mathrm{CuCl} /$ glass, respectively, in the following. Three types of cryostats were used depending on the temperature range: a ${ }^{4} \mathrm{He}$ continuous flow cryostat $(T$ $\geqslant 2 \mathrm{~K}$ ), a liquid- ${ }^{4} \mathrm{He}$ immersion cryostat $(1.5 \mathrm{~K} \leqslant T \leqslant 2 \mathrm{~K})$, and a ${ }^{3} \mathrm{He}$ cryostat whose temperature is monitored by a calibrated $\mathrm{RuO}_{2}$ thermometer $(T \leqslant 1.5 \mathrm{~K})$.

Absorption spectra of the samples at $2 \mathrm{~K}$ are shown at the upper part of Fig. 1 by solid lines. The lowest $Z_{3}$-exciton absorption band is shifted toward the higher-energy side from the bulk value $(3.202 \mathrm{eV})$ due to the quantum size effect. The average radius of the spherical $\mathrm{CuCl}$ QD's in glass is estimated to be $2.0 \mathrm{~nm}$ from the peak position of the 


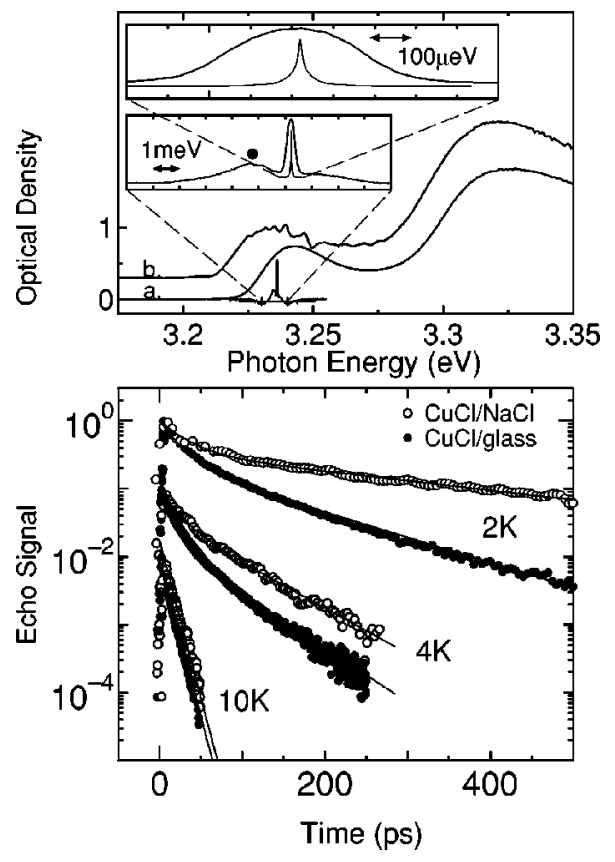

FIG. 1. Upper: Absorption spectra of $\mathrm{CuCl}$ quantum dots at $2 \mathrm{~K}$, (a) $\mathrm{CuCl} /$ glass and (b) $\mathrm{CuCl} / \mathrm{NaCl}$. In the insets, a solid curve shows the PSHB spectrum of $\mathrm{CuCl} /$ glass at $2 \mathrm{~K}$ with expanded scale. The very sharp Lorentzian curve drawn by a thin line represents the Fourier transform of the echo decay curve. Lower: Temperaturedependent echo signal of $\mathrm{CuCl}$ quantum dots measured by the heterodyne-detected accumulated photon echo. The solid circles and the open circles correspond to data for $\mathrm{CuCl} / \mathrm{glass}$ and $\mathrm{CuCl} / \mathrm{NaCl}$, respectively. The signals were similar at $10 \mathrm{~K}$ between the two samples, but obvious differences were observed at $2 \mathrm{~K}$. Solid lines represent double exponential decay (single exponential decay for $10 \mathrm{~K}$ data). The decay time constants of the slow component were $310 \mathrm{ps}, 117 \mathrm{ps}, 65 \mathrm{ps}, 55 \mathrm{ps}, 9.5 \mathrm{ps}$, and $9.5 \mathrm{ps}$ from top to bottom.

$Z_{3}$ exciton. The wavy structures seen in the $Z_{3}$-exciton absorption region of $\mathrm{CuCl} / \mathrm{NaCl}$ can be explained by the steplike increase of the size of cubic shape QD's. ${ }^{15,16}$ Examples of the accumulated photon echo signal of $\mathrm{CuCl}$ quantum dots are shown at the lower part of Fig. 1 for three temperatures. The photon energy and the excitation density were $3.23 \mathrm{eV}$ and $100 \mathrm{pJ} / \mathrm{cm}^{2}$. In the heterodyne-detection scheme, the dephasing time is obtained as twice the echo decay time $(\tau)$. Then the homogeneous width is given by $\Gamma_{h}=\hbar / \tau$. At low temperatures, the echo signal often showed double exponential decay. In this case, $T_{2}$ was obtained from the slower decay component. The fast component whose decay time is $10-30 \mathrm{ps}$ is most probably caused by a phonon sideband, and does not correspond to the broadening of the zero-phonon line. The first point to notice is that the homogeneous linewidth obtained in our experiment is extremely sharp. At the upper part of Fig. 1 and its insets, the PSHB spectrum of $\mathrm{CuCl} /$ glass is shown by a solid line. The homogeneous linewidth is evaluated from half of the FWHM of the spectral hole. It is known that the Fourier cosine transformation of the heterodyne-detected accumulated photon echo signal gives the PSHB spectrum. ${ }^{17}$ The Fourier transformed curve of the echo data at $2 \mathrm{~K}$ is represented in the inset by a fine solid line. We can see that the echo signal gives a much sharper linewidth than the directly measured PSHB line. An-

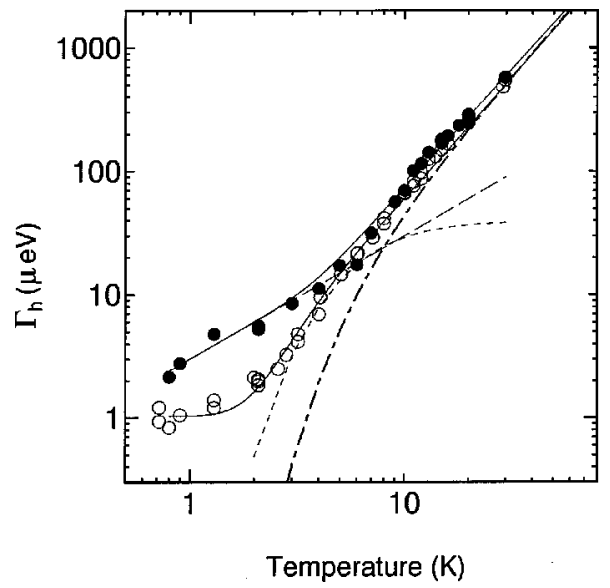

FIG. 2. Solid circles represent the homogeneous width of the $Z_{3}$ exciton of $\mathrm{CuCl} / \mathrm{glass}$ as a function of temperature. Open circles show the homogeneous width of $\mathrm{CuCl} / \mathrm{NaCl}$. Above $5 \mathrm{~K}$, the temperature dependence of the two samples are similar, while a remarkable difference can be seen below $5 \mathrm{~K}$. Long-dashed and shortdashed curves represent the temperature-dependent TLS contribution of $\mathrm{CuCl} / \mathrm{glass}$ and $\mathrm{CuCl} / \mathrm{NaCl}$, respectively.

other important point is that the echo signal is different for the two samples at low temperature. At $10 \mathrm{~K}$, the two signals are almost the same, but the difference becomes clear with the decrease in temperature. At $2 \mathrm{~K}$, the echo signal of $\mathrm{CuCl} / \mathrm{NaCl}$ shows more than twice longer decay time than that of $\mathrm{CuCl} / \mathrm{glass}$.

Homogeneous linewidth obtained from these data are plotted in Fig. 2 as a function of temperature. In this figure, solid (open) circles represent the homogeneous width of $\mathrm{CuCl} / \mathrm{glass}(\mathrm{CuCl} / \mathrm{NaCl})$. In comparison to previous works in the spectral domain, $, 8,9,18,19$ our data give a very small homogeneous width at low temperatures as mentioned before. For example, the linewidth of $\mathrm{CuCl}$ quantum dots embedded in a $\mathrm{NaCl}$ crystal measured by PSHB was $70 \mu \mathrm{eV}$ at $2 \mathrm{~K}^{19}$ which gave the narrowest homogeneous width of the $Z_{3}$ exciton of $\mathrm{CuCl}$ quantum dots so far. However, it is found to be $2 \mu \mathrm{eV}$ in our measurement, which is smaller by an order of magnitude. In the PSHB case, the spectral resolution of the spectrometer is comparable to the homogeneous width. Furthermore, the spectral diffusion occurring in the long interval from the hole-burning process to the absorption measurement may affect the experimental results. So we believe that we have reliably measured homogeneous width by accumulated photon echo method, though there remains a problem that possible spectral diffusion during the accumulation process (several seconds) may broaden the homogeneous spectrum. Observed homogeneous width reached $1 \mu \mathrm{eV}$ at low temperature. This is the smallest homogeneous width of a quantum dot system to our knowledge. The value is narrower than the homogeneous width of excitonic polaritons in the bulk crystal by an order of magnitude. ${ }^{6,7}$ This extreme narrowing of the homogeneous width of confined excitons in quantum dots is considered to come from the spatial confinement of the exciton wave function and that of the acoustic phonons in quantum dots.

The temperature dependence of the homogeneous width of both samples were similar above $5 \mathrm{~K}$, but we can see an obvious difference between them below this temperature. 
For $\mathrm{CuCl} / \mathrm{NaCl}$, the homogeneous width continues to decrease rapidly below $5 \mathrm{~K}$ and approaches a lower limit. On the other hand, the homogeneous width of the $\mathrm{CuCl} / \mathrm{glass}$ changes its slope at $5 \mathrm{~K}$ and shows almost linear temperature dependence in the low-temperature region. Similar behavior of $\mathrm{CuCl} /$ glass that we recognize also in the data of Ref. 13 (the authors did not discuss it) provides us with an independent check for our data for $\mathrm{CuCl} /$ glass. Then we have tested many $\mathrm{CuCl} / \mathrm{NaCl}$ samples to confirm that the difference of the temperature dependence at low temperatures was not a sample-dependent effect, but an essential and intrinsic one. It indicates that the matrix surely affects the dephasing rate of the exciton confined in quantum dots in the low-temperature region.

Let us discuss the origin of the temperature-dependent homogeneous broadening of confined excitons. As one mechanism that determines the homogeneous broadening at high temperature, interaction of the confined exciton and the confined phonon in quantum dots is appropriate. LO-phonon scattering is not important in the temperature range, but increases rapidly above $50 \mathrm{~K} .{ }^{19} \mathrm{~A}$ confined acoustic phonon is experimentally observed for $\mathrm{CuCl}$ quantum dots by PSHB. ${ }^{20}$ For our sample, as seen in the inset of Fig. 1, a sideband due to the confined acoustic phonon marked by a solid circle was clearly observed, which was separated from the zero-phonon line by $1.6 \mathrm{meV}$. For the high-temperature region, experimental data for both samples having $\mathrm{NaCl}$ and glass matrix can be fitted by assuming that the homogeneous width is determined by a two-phonon Raman process where absorption and emission of a phonon simultaneously occur and whose probability is proportional to $n(n+1)$, where $n$ represents the occupation number of the confined acoustic phonon. Then the temperature dependence of the homogeneous broadening is given by $\Gamma_{h}(T) \propto \sinh ^{-2}(\hbar \omega / 2 k T)$, where $\hbar \omega$ is the energy of the lowest confined phonon mode. A dotdashed line in Fig. 2 shows the contribution of the confined acoustic phonon for $\mathrm{CuCl} / \mathrm{glass}$.

Obviously, one cannot explain the observed matrixdependent temperature dependence in a low-temperature region by this phonon mode. We should consider another contribution that is related to the matrix of the quantum dots. One possible dephasing process is coupling to a two-level system (TLS) (Ref. 21) which had been used to explain the anomalous temperature dependence of the homogeneous linewidth of impurity atoms in a glass matrix or dye molecules in a polymer. The basic concept of TLS is that there are double potential well systems in glass where quantummechanical tunneling can occur. Temperature-dependent line broadening by the interaction with TLS is expressed in the form of $\cosh ^{-2}(\hbar \omega / 2 k T)$, here $\hbar \omega$ is the characteristic energy of TLS. The randomness in glass is considered to make the energy of the TLS distributed over a wide range. Therefore, broadening due to TLS is often approximated as $T^{\alpha}$ $(1<\alpha<2){ }^{21}$ We can reproduce the experimental data for $\mathrm{CuCl} / \mathrm{glass}$ by the sum of the contributions from the confined acoustic phonon and the T-linear TLS processes as shown in Fig. 2 by a solid line on the open circles. On the other hand, the experimental data for $\mathrm{CuCl} / \mathrm{NaCl}$ can be well reproduced if the energy of TLS - they exist not only in glassy systems but also in crystals ${ }^{22}$-is concentrated on a characteristic energy $\hbar \omega^{\prime}$. Then the contribution of TLS is directly propor- tional to $\cosh ^{-2}\left(\hbar \omega^{\prime} / k T\right)$. Finally, the temperature dependence of the homogeneous width of $\mathrm{CuCl} / \mathrm{NaCl}$ is represented by

$$
A \sinh ^{-2}(\hbar \omega / 2 k T)+B \cosh ^{-2}\left(\hbar \omega^{\prime} / 2 k T\right)+\Gamma_{0} .
$$

A solid curve on the open circles in Fig. 2 shows this temperature dependence in the case of $\hbar \omega^{\prime}=1 \mathrm{meV}$. The fitting by the TLS model agrees well with the experimental results for both $\mathrm{CuCl} / \mathrm{glass}$ and $\mathrm{CuCl} / \mathrm{NaCl}$. These satisfactory results lead to the conclusion that the matrix makes a difference in the temperature dependence of the homogeneous width of excitons in quantum dots through the difference of the energy distribution of TLS.

It is important to compare $T_{2}$ at the low-temperature limit and the longitudinal relaxation time $T_{1}$. If the pure dephasing, which does not involve population relaxation of excitons, disappears at low temperature, the transverse relaxation rate should equal half the longitudinal relaxation rate. Experimentally, $T_{1}$ was obtained by the luminescence decay time at very low excitation power. The luminescence showed single-exponential decay with a decay time of $3.56 \mathrm{~ns}$ for $\mathrm{CuCl} / \mathrm{NaCl}$. The excitation density was the same as that in the photon echo measurements. In $\mathrm{CuCl} /$ glass, on the other hand, the luminescence lifetime was 600 ps. The natural widths corresponding to these $T_{1}$ are $0.19 \mu \mathrm{eV}(\mathrm{CuCl} / \mathrm{NaCl})$ and $1.1 \mu \mathrm{eV}(\mathrm{CuCl} / \mathrm{glass})$, respectively. The observed lower limit of the homogeneous width of $\mathrm{CuCl} / \mathrm{glass}$ is $2 \mu \mathrm{eV}$ at $0.7 \mathrm{~K}$, almost the same as the natural width. On the other hand, the lowest value of the homogeneous width of the $\mathrm{CuCl} / \mathrm{NaCl}$ is $1 \mu \mathrm{eV}$, which is five times larger than the natural width. Therefore, another low-energy dephasing mechanism may exist in the $\mathrm{CuCl} / \mathrm{NaCl}$. The origin of this process is not clear, but absence of temperature dependence indicates that it is impurity or surface scattering. For $\mathrm{CuCl} / \mathrm{glass}$, this unknown mechanism is hidden by the fast longitudinal relaxation rate due to nonradiative population relaxation. Luminescence decay of $\mathrm{CuCl} / \mathrm{glass}$ did not change in the temperature range of this study. Therefore, it is sure that the linear temperature dependence of the homogeneous width is not due to the change of $T_{1}$. At the lowtemperature limit, the confined acoustic phonon and the TLS are frozen because of their finite energy and do not contribute the dephasing of confined exitons in $\mathrm{CuCl}$ quantum dots. The remaining dephasing processes are impurity or surface scattering and/or nonradiative relaxation that are also less efficient in the quantum dots than the bulk crystal, because excitons ideally confined in a small space have less probability to interact with impurities and surface than excitonic polariton propagating in the bulk crystal.

In conclusion, the temperature-dependent homogeneous linewidth of excitons confined in $\mathrm{CuCl}$ quantum dots embedded in a alminoborosilicate glass and in a $\mathrm{NaCl}$ crystal were investigated by the heterodyne-detected accumulated photon echo technique. Under very weak excitation condition and at low temperatures, the photon echo signal shows a long decay time corresponding to a very sharp linewidth. Especially, at $1 \mathrm{~K}$, the exciton linewidth of $\mathrm{CuCl} / \mathrm{NaCl}$ was $1 \mu \mathrm{eV}$, which is the smallest reported value of the linewidth of quantum dots and much narrower than that of the bulk crystal. Above $5 \mathrm{~K}$, broadening of the homogeneous linewidth is explained 
by the scattering by the confined phonon. The temperature dependence of the homogeneous width was different between $\mathrm{CuCl} / \mathrm{NaCl}$ and $\mathrm{CuCl} /$ glass in the low-temperature region below $5 \mathrm{~K}$. The different temperature dependence is related to the difference of the energy distribution of TLS that determines the line broadening below $5 \mathrm{~K}$.

We wish to express our gratitude to Professor Y. Ootuka, Dr. M. Morishita, and M. Furuya of the University of Tsukuba for their technical guidance and support in the lowtemperature measurements. We acknowledge helpful discus- sions with Dr. T. Mishina and Dr. T. Okuno of the University of Tsukuba. Thanks are due to Dr. S. V. Nair for reading the entire text in its original form. This work was supported in part by the Research for the Future Program No. JSPSRFTF97P00106 from the Japan Society for the Promotion of Science, Grant-in-Aid Scientific Research No. 10554011 from the Ministry of Education, Science, Sports, and Culture of Japan, the Research Fellowships of the Japan Society for the Promotion of Science for Young Scientists, and the Project Research of the University of Tsukuba.
${ }^{1}$ See, for example, L. Bányai and S. W. Koch, in Semiconductor Quantum Dots (World Scientific, Singapore, 1993).

${ }^{2}$ K. Naoe, L. G. Zimin, and Y. Masumoto, Phys. Rev. B 50, 18 200 (1994).

${ }^{3}$ V. Jungnickel and F. Henneberger, J. Lumin. 70, 238 (1996).

${ }^{4}$ M. Nirmal et al., Nature (London) 383, 802 (1996).

${ }^{5}$ M. Kalm and Ch. Uihlein, Phys. Status Solidi B 87, 575 (1978).

${ }^{6}$ Y. Masumoto, S. Shionoya, and T. Takagahara, Phys. Rev. Lett. 51, 923 (1983).

${ }^{7}$ F. Vallée, F. Bogani, and C. Flytzanis, Phys. Rev. Lett. 66, 1509 (1991)

${ }^{8}$ T. Wamura, Y. Masumoto, and T. Kawamura, Appl. Phys. Lett. 59, 1758 (1991).

${ }^{9}$ T. Itoh and M. Furumiya, J. Lumin. 48\&49, 704 (1991).

${ }^{10}$ D. Gammon et al., Science 273, 87 (1996).

${ }^{11}$ S. A. Empedocles, D. J. Norris, and M. G. Bawendi, Phys. Rev. Lett. 77, 3873 (1996).

${ }^{12}$ S. Saikan, K. Uchikawa, and H. Ohsawa, Opt. Lett. 16, 10 (1991).
${ }^{13}$ R. Kuribayashi et al., Phys. Rev. B 57, 15084 (1998).

${ }^{14} \mathrm{M}$. Ikezawa and Y. Masumoto, in Proceedings of the 24th International Conference on the Physics of Semiconductors, Jerusalem, 1998, edited by D. Gershoni (World Scientific, Singapore, 1999).

${ }^{15}$ T. Itoh et al., J. Lumin. 60\&61, 396 (1994).

${ }^{16}$ N. Sakakura and Y. Masumoto, Phys. Rev. B 56, 4051 (1997).

${ }^{17}$ S. Saikan et al., Phys. Rev. B 38, 7777 (1988).

${ }^{18}$ T. Kuroda et al., Phys. Rev. B 55, 16041 (1997).

${ }^{19}$ Y. Masumoto, T. Kawazoe, and N. Matsuura, J. Lumin. 76-77, 189 (1998)

${ }^{20}$ S. Okamoto and Y. Masumoto, J. Lumin. 64, 253 (1995); J. Zhao and Y. Masumoto, Phys. Rev. B 60, 4481 (1999).

${ }^{21}$ See, for example, R. M. Macfarlane and R. M. Shelby, J. Lumin. 36, 179 (1987); D. L. Huber, in Dynamical Processes in Disordered Systems, edited by W. M. Yen (Trans. Tech. Aedermannsdorf, Switzerland, 1989).

${ }^{22}$ G. P. Flinn et al., Phys. Rev. B 49, 5821 (1994). 